\title{
SENSITIVITY OF JOSEPHSON-EFFECT MILLIMETER-WAVE RADIOMETER $\left({ }^{*}\right)$
}

\author{
H. OHTA $(*)^{\dagger}$, M. J. FELDMAN, P. T. PARRISH and R. Y. CHIAO
}

Department of Physics, University of California, Berkeley, California 94720, USA

\begin{abstract}
Résumé. - On calcule la température de bruit et la température minimum détectable d'une jonction Josephson soumise à un rayonnement millimétrique en utilisant l'équation de FokkerPlanck.

La température de bruit obtenue peut être très voisine de la température ambiante.

Un récepteur pour radiotélescope à très large bande utilisant une jonction Josephson peut avoir une température minimum détectable inférieure à celle d'un maser à ondes progressives.
\end{abstract}

Abstract. - The noise temperature and the minimum detectable temperature of a Josephson junction in video detection of microwave and millimeter-wave radiation has been calculated. We use the well-known method based on a Fokker-Planck equation. The noise temperature can be very close to ambient temperature. Because its predetection bandwidth is very wide, a Josephsoneffect radio telescope receiver can have a minimum detectable temperature better than that of a traveling-wave maser.

We apply monochromatic radiation to a Josephson junction to determine the frequency dependence of the response. We use a phenomenological model for a Josephson junction, with signal radiation and current noise, described by the equations :

$$
\begin{gathered}
\hbar \frac{\partial \varphi}{\partial t}=2 \mathrm{eV} \\
C \frac{\mathrm{d} V}{\mathrm{~d} t}+\frac{V}{R}+I_{\mathrm{m}} \sin \varphi=I+i \cos (\omega t+\theta)+i_{\mathrm{n}}(t) .
\end{gathered}
$$

These are standard symbols and are given in reference [1]. $R$ is the parallel combination of the junction resistance and the source resistance. The noise current has a white spectum

$$
<i_{\mathrm{n}}(t) i_{\mathrm{n}}\left(t^{\prime}\right)>=\frac{2 k T I}{\bar{V}} \delta\left(t-t^{\prime}\right) .
$$

A very important assumption is that the capacitance is small

$$
R C \ll \frac{\hbar}{2 \mathrm{e} I_{\mathrm{m}} R} .
$$

We treat the radiation as a perturbation. The solution of the unperturbed equation without radiation or noise is the voltage-current curve

$$
\left(\frac{\bar{V}_{0}}{R}\right)^{2}=I_{0}^{2}-I_{\mathrm{m}}^{2} \quad\left(I_{0}>I_{\mathrm{m}}\right)
$$

(*) Work supported partially by National Science Foundation Grant GP 38586, National Aeronautics and Space Administration Grant NGL 05-003-272, and † National Radio Astronomy Observatory under Prime Contract no. NSF-C450, contract no. AUI-1011.
A voltage increment, $V_{1}$, near the first radiationinduced step is given by :

$$
V_{1}=\left(\frac{\hbar}{2 \mathrm{e}}\right) \frac{\partial \varphi_{1}}{\partial t}=V-\frac{\hbar \omega}{2 \mathrm{e}}-R i \cos (\omega t+\theta) .
$$

As in reference [1] we find that $V_{1}$ obeys the equation

$$
C_{1} \frac{\mathrm{d} V_{1}}{\mathrm{~d} t}+\frac{V_{1}}{R_{\mathrm{D}}}+i_{1} \sin \varphi_{1}=i_{\mathrm{b}}+i_{\mathrm{n}}(t)
$$

where

$$
\begin{gathered}
i_{\mathrm{b}}=I-I_{0}, \quad i_{1}=\frac{i}{2}\left(1+\xi^{2}\right)^{-1 / 2} \\
\xi=\frac{\hbar \omega}{2 \mathrm{e} I_{\mathrm{m}}} R, \quad R_{\mathrm{D}} C_{1}=R C
\end{gathered}
$$

and where from eq. (4)

$$
\begin{aligned}
& R_{\mathrm{D}} \equiv \frac{\mathrm{d} \bar{V}_{0}}{\mathrm{~d} I_{0}}=R\left(1+\frac{1}{\xi^{2}}\right)^{1 / 2} \\
& \frac{R I}{\overline{\bar{V}}} \simeq \frac{R I_{0}}{\bar{V}_{0}}=\frac{R_{\mathrm{D}}}{R} .
\end{aligned}
$$

Comparing eq. (1) and eq. (5), we see that $i_{1}$ is the zero-noise one-half height of the first radiation-induced step.

Before going into a Fokker-Planck equation, we wish to examine the connection between $2 D$, the second order moment of the fluctuating phase $\varphi_{1}$, and $\tau$, the time taken for a single measurement. $\tau$ also explains the post-detection bandwidth. $D$, as we shall see below, is closely related to the predetection bandwidth. In fact, for a Lorentzian line shape, $D$ is equal to the pre-detection bandwidth. 
Eq. (2) and (5) give the correlation function of the fluctuating voltage

$$
<\delta V_{1}(t) \delta V_{1}\left(t^{\prime}\right)>=\frac{k T R_{\mathrm{D}} I}{C_{1} \bar{V}} \exp \left(-\frac{\left|t-t^{\prime}\right|}{R C}\right) .
$$

Using eq. (1) and (8) we see that [2]

$$
\begin{aligned}
2 D & =\frac{1}{\tau}\left\langle\left(\varphi_{1}(\tau)-\varphi_{1}(0)\right)^{2}\right\rangle \\
& =\frac{8 \mathrm{e}^{2} \tau}{\hbar^{2}} \int_{0}^{\infty} g(t)<\delta V_{1}(0) \delta V_{1}(t)>\mathrm{d} t \\
& =\frac{8 \mathrm{e}^{2} k T R_{\mathrm{D}}^{2} I}{\hbar^{2} \bar{V}}\left[1-\frac{1}{\alpha}\left(1-\mathrm{e}^{-\alpha}\right)\right]
\end{aligned}
$$

where we have defined

$$
\begin{gathered}
g(t)=\left\{\begin{array}{cc}
\frac{\tau-|t|}{\tau^{2}} & |t|<\tau \\
0 & |t|>\tau
\end{array}\right. \\
\alpha=\frac{\tau}{R C} .
\end{gathered}
$$

For measurement times much larger than the junction response time, $\alpha \gg 1$, the first term of eq. (9) is dominant. For very short measurement times, $\alpha \ll 1$, the line width is Gaussian and equal to

$$
\frac{2 \mathrm{e}}{\hbar} \sqrt{\frac{k T R_{\mathrm{D}} I}{C_{1} \bar{V}}},
$$

which is larger than in eq. (9). This phenomenon is just an example of « motional narrowing due to thermal fluctuations » [3], [4].

To get a stationary solution of the Fokker-Planck equation we assume $\alpha \gg 1$ and thus

$$
D=\frac{4 \mathrm{e}^{2} k T R_{\mathrm{D}}^{2} I}{\hbar^{2} \bar{V}} .
$$

We get a Fokker-Planck equation with the first and the second order moments of $\varphi_{1}$ requiring the conservation of probability

$$
\frac{\partial W}{\partial t}=\frac{\partial}{\partial \varphi_{1}}\left[\frac{2 \mathrm{e} R_{\mathrm{D}}}{\hbar}\left(i_{1} \sin \varphi_{1}-i_{\mathrm{b}}\right) W+D \frac{\partial W}{\partial \varphi_{1}}\right] .
$$

In the field of radio engineering, Stratonovich [5] has solved this differential equation in examining the synchronization of two oscillators in the presence of noise. In a similar way, Haken et al. [6] studied laser noise in the phase-locking region. The effect of noise on Josephson junctions has been examined by several authors in the same way [7], [8]. The solution of the Fokker-Planck equation is

$$
\bar{V}_{1}=\frac{\frac{R_{\mathrm{D}} i_{0}}{2} \operatorname{sh} \frac{\pi i_{\mathrm{b}}}{i_{0}}}{\int_{0}^{\pi / 2} \operatorname{ch}\left(\frac{2 i_{\mathrm{b}} \theta}{i_{0}}\right) I_{0}\left(\frac{2 i_{1}}{i_{0}} \cos \theta\right) \mathrm{d} \theta}
$$

where $I_{0}(z)$ is the modified Bessel function and

$$
i_{0}=\frac{2 \mathrm{e} k T R_{\mathrm{D}} I}{\hbar \bar{V}}=\frac{\hbar D}{2 \mathrm{e} R_{\mathrm{D}}}
$$

is the characteristic noise current [9].

We are interested in considering very weak radiation, the radiation-induced step is almost completely washed out by the noise. In this case, $i_{0} \gg i_{1}$, so that we can expand the Bessel function in eq. (12), keeping the first two terms to give

$$
\bar{V}_{1}=R_{\mathrm{D}} i_{\mathrm{b}}-\frac{i_{\mathrm{b}}}{i_{\mathrm{b}}^{2}+i_{0}^{2}} \frac{R_{\mathrm{D}} i_{1}^{2}}{2} \quad\left(i_{0} \gg i_{1}\right) .
$$

This equation is illustrated in figure 1 . In the small bias current limit eq. (13) reduces to eq. (3.10) of reference [7] for the case of small $a_{2}\left(=i_{1} / i_{0}\right)$. Eq. (13) is consistent with eq. (30) of reference [10] which was derived by a slightly different approach for a constant voltage source.

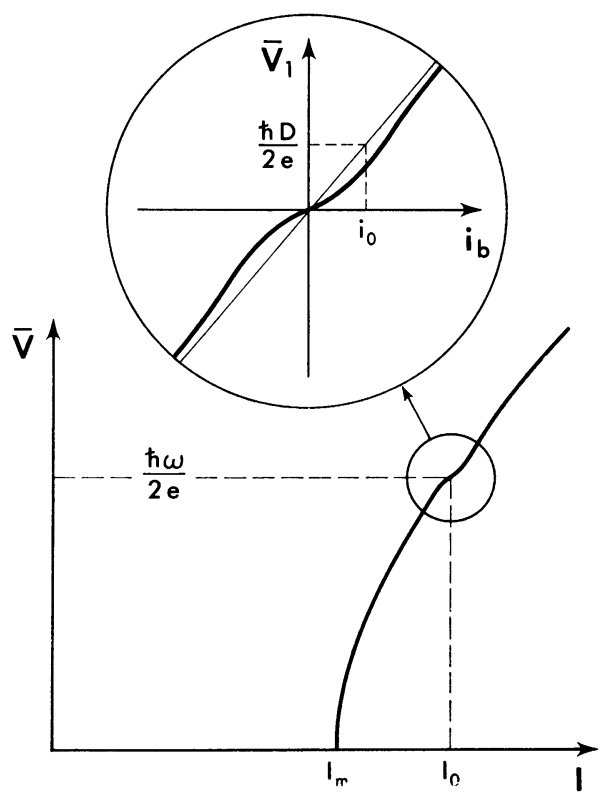

Fig. 1. - The voltage-current characteristic with a small radiation-induced step. The expanded portion of the curve, inside the circle, is given by eq. (13). The effect of the radiation has been exaggerated.

The coupling coefficient $\kappa$, the ratio of the absorbed power to the power of the incident radiation is given by

$$
\kappa=\frac{\mathrm{d}\left(R_{\mathrm{D}} i_{1}^{2} / 2\right)}{\mathrm{d} P}=\frac{\left(R_{\mathrm{D}} / 4 Z\right)}{1+\xi^{2}}
$$

where $Z$ is the characteristic impedance of the cavity. Eq. (13) immediately gives the responsivity of the junction to the radiation,

$$
s=\frac{\mathrm{d} \bar{V}_{1}}{\mathrm{~d} P}=\frac{\kappa \hbar \bar{V}}{4 \mathrm{e} k T R_{\mathrm{D}} I} \cdot \frac{2 i_{0} i_{\mathrm{b}}}{i_{\mathrm{b}}^{2}+i_{0}^{2}} .
$$


This responsivity is maximum at a bias current $i_{\mathrm{b}}$ equal to the noise current $i_{0}$. We see that the Josephson frequency of the optimum bias voltage is not equal to the radiation frequency, and in fact

$$
\left|\omega-\frac{2 \mathrm{e} \bar{V}}{\hbar}\right|=D \text {. }
$$

We are now ready to calculate the minimum detectable temperature of a Josephson-effect radio telescope receiver. We have a spectral power gain from eq. (11)

$$
H(v)=\frac{\mathrm{e} D}{2 \pi^{2} \hbar} \cdot \frac{1}{v^{2}+(D / 2 \pi)^{2}} .
$$

The pre-detection bandwidth $B$ is then [11]

$$
B=\frac{\left(\int_{-\infty}^{\infty} H(v) \mathrm{d} v\right)^{2}}{\int_{-\infty}^{\infty} H(v)^{2} \mathrm{~d} v}=D
$$

The frequency response of an ideal integrator is given by

$$
G(v)=\left(\frac{\sin \pi \tau v}{\pi \tau v}\right)^{2}
$$

whereby the post-detection bandwidth $B_{\mathrm{L}}$ is [11]

$$
B_{\mathrm{L}}=\frac{1}{G(0)} \int_{0}^{\infty} G(v) \mathrm{d} v=\frac{1}{2 \tau} .
$$

Note that $G(v)$ is the Fourier transform of $g(t)$ of eq. (10). The noise power from the receiver is

$$
\begin{aligned}
N=\frac{1}{R_{\mathrm{D}}} & \iint_{-\infty}^{\infty} G(v) H(v-\sigma) w(v-\sigma) H(\sigma) w(\sigma) \mathrm{d} v \mathrm{~d} \sigma \\
& \simeq \frac{2 k T R_{\mathrm{D}} I B_{\mathrm{L}}}{\bar{V}}\left[1-\frac{1}{2 D \tau}\left(1-\mathrm{e}^{-2 D \tau}\right)\right]
\end{aligned}
$$

where we used another Fourier transform

$$
\begin{aligned}
w(v) & =\int_{-\infty}^{\infty}\left\langle\delta V_{1}(0) \delta V_{1}(t)>\mathrm{e}^{-i \omega t} \mathrm{~d} t\right. \\
& =\frac{2 k T R_{\mathrm{D}}^{2} I}{\bar{V}} \frac{1}{1+(R C \omega)^{2}} .
\end{aligned}
$$

We measure the decrease in the dynamic resistance due to thermal radiation. In the same way we have the signal power from the receiver

$$
S=\frac{\left(\Delta \bar{V}_{1}\right)^{2}}{2 R_{\mathrm{D}}} \text {. }
$$

The condition that the $\mathrm{SN}$ ratio is unity gives the minimum detectable power

$$
\begin{aligned}
P_{\min } & =\frac{1}{s} \sqrt{\frac{4 k T R_{\mathrm{D}}^{2} I B_{\mathrm{L}}}{\bar{V}}} \\
& =\frac{4 k T R_{\mathrm{D}} I}{\kappa \bar{V}} \sqrt{B B_{\mathrm{L}}} .
\end{aligned}
$$

Defining the noise temperature, $T_{\mathrm{N}}$, the minimum detectable temperature, $\Delta T$, and the noise equivalent power, $N E P$, in the standard way [13], [14], [15]

$$
\begin{aligned}
P_{\min } & =k T_{\mathrm{N}} \sqrt{2 B B_{\mathrm{L}}}=N E P \sqrt{2 B_{\mathrm{L}}}=k \Delta T B \\
T_{\mathrm{N}} & =\frac{2 \sqrt{2}}{\kappa}\left(1+\frac{1}{\xi^{2}}\right) T \\
\Delta T & =\frac{T_{\mathrm{N}}}{\sqrt{B \tau}}=T_{\mathrm{N}} \sqrt{\frac{2 B_{\mathrm{L}}}{B}} \\
& =\frac{\sqrt{2}}{\kappa}\left(1+\frac{1}{\xi^{2}}\right)^{1 / 4} \frac{\hbar}{\mathrm{e}} \sqrt{\frac{T}{k R \tau}} .
\end{aligned}
$$

Eq. (22) shows that the noise temperature can be very close to the ambient temperature when the coupling coefficient is near unity and the normalized Josephson frequency $\xi$ is large. This is an almost ideal passive detector. We calculate a theoretical example using very reasonable experimental parameters. Assuming

$$
\begin{array}{ll}
R=12.5 \Omega, & Z=50 \Omega, \\
I_{\mathrm{m}}=40 \mu \mathrm{A}, & T=2 \mathrm{~K}, \\
\tau=1 \mathrm{~s}, & \lambda=2 \mathrm{~mm},
\end{array}
$$

we have

$$
\begin{aligned}
\xi & =0.62 \\
\kappa & =0.086 \\
R_{\mathrm{D}} & =23.7 \Omega
\end{aligned}
$$

and

$$
\begin{aligned}
s & =1.42 \times 10^{5} \mathrm{~V} / \mathrm{W} \\
N E P & =4.84 \times 10^{-16} \mathrm{~W} / \sqrt{\mathrm{Hz}} \\
T_{\mathrm{N}} & =238 \mathrm{~K} \\
B & =2.18 \times 10^{10} \mathrm{~Hz} \\
\Delta T & =1.61 \times 10^{-3} \mathrm{~K} .
\end{aligned}
$$

The experimentally established best NEP is $5 \times 10^{-15} \mathrm{~W} / \sqrt{\mathrm{Hz}}$ [16]. If either the coupling is very poor or the product $I_{\mathrm{m}} R_{\mathrm{J}}$ of the Josephson junction is much smaller than the energy gap of the superconductor, we cannot realize this sensitivity. Adjusting the parameters in any other way does not change these sensitivity figures very much. Note that the admittedly theoretical minimum detectable temperature is better than that of existing travelingwave masers [17]. The figure of merit of a radio telescope receiver is equal to the inverse of its minimum detectable temperature assuming the unity integration time. Although masers have a lower noise temperature, their pre-detection bandwidth is much narrower than that of Josephson junctions. Josephson-effect video detectors would be advantageous in the measurement of continuum spectra.

Acknowledgments. - The authors gratefully acknowledge Professor C. H. Townes and Dr. S. Weinreb for their encouragement and long support. 


\section{References}

[1] Oнta, H., J. Appl. Phys. 43 (1972) 5161.

[2] KuBo, R., Statistical Mechanics (North-Hall and Publishing Company-Amsterdam) 1967, p. 400.

[3] Abragam, A., The Principles of Nuclear Magnetism (Oxford at the Clarendon Press), 1961, Chapter 10.

[4] Scalapino, D. J., Proceedings of the Symposium on the Physics of Superconducting Devices, University of Virginia, Charlottesville, 1967 (Customer Services Clearinghouse, US Department of Commerce, Springfield, Virginia 22151).

[5] Stratonovich, R. L., Topics in the Theory of (Random Noise, (Gordon and Breach, New York) 1967, Chapter 9., vol. 2.

[6] Haken, H., Sauermann, H., Schmid, Ch. and Vollmer, H. D., Z. Phys. 206 (1967) 369.

[7] Stephen, M. J., Phys. Rev. 186 (1969) 393 ; Phys. Rev. 182 (1969) 531.
[8] Ambegaokar, V. and Halperin, B. I., Phys. Rev. Lett. 22 (1969) 1364.

[9] Clarke, J., Phys. Rev. 413 (1971) 2963.

[10] Kalashnik, L. I., Kislov, A. M., Kulik, I. O., Livshits, E. M., Maslov, K. V. and Motornaya, A. A., Soviet Physics. Technical Physics 17 (1972) 1029.

[11] Kraus, J. D., Radio Astronomy (McGraw-Hill, New York) 1966, Chapter 7 by Tiuri, M. E.

[12] Bateman, H., Tables of Integral Transformations (McGrawHill, New York) 1 (1954) 19.

[13] Dicke, R. H., Rev. Sci. Instrum. 17 (1946) 268.

[14] Selove, W., Rev. Sci. Instrum. 25 (1954) 120.

[15] Giordmaine, J. A., Alsop, L. E., Mayer, C. H., Townes, C. H., Proc. IRE 47 (1959) 1062.

[16] Kanter, H. and Vernon, F. L., Jr., J. Appl. Phys. 43 (1972) 3174.

[17] Siegman, A. E., Microwave Solid-State Masers (McGrawHill, New York) 1964, Chapter 9. 